\title{
The role of serum inflammatory markers, albumin, and hemoglobin in predicting the diagnosis in patients admitted to the emergency department with a pre-diagnosis of COVID-19
}

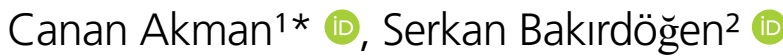

\begin{abstract}
SUMMARY
OBJECTIVE: Serum inflammatory markers and albumin levels provide an assumption for the severity of COVID-19 infection. Our objective was to investigate the determinant role of serum inflammatory markers, albumin, and hemoglobin ( $\mathrm{Hb})$ in predicting the diagnosis in patients with a pre-diagnosis of COVID-19.

METHODS: Demographic findings, complete blood count and serum biochemical values of the patients analyzed. RESULTS: Of the patients included in the study, 48 were COVID (+) and 253 were COVID (-). Statistically significant difference was found in terms of hemoglobin, mean platelet volume, and monocyte/eosinophil ratio.

CONCLUSIONS: The levels of serum albumin, hemoglobin, monocyte/eosinophil ratio, and mean platelet volume can be predictive factors for diagnosis in patients with COVID-19.

KEYWORDS: Hypoalbuminemia. Hemoglobins. Mean platelet volume. Monocytes. Eosinophils. Coronavirus infections. Emergency service, hospital.
\end{abstract}

\section{INTRODUCTION}

The COVID-19 infection (2019-nCoV), which first occurred in China and spread all over the world in December 2019, was accepted as a pandemic by the World Health Organization ${ }^{1}$. COVID-19 infection causes high morbidity and mortality in patients due to the risks of severe pneumonia, ARDS (Adult Respiratory Distress Syndrome), acute kidney injury, and acute heart failure ${ }^{2}$. It has been observed that there is a relationship between hypoalbuminemia and severe COVID-19 infection ${ }^{3,4}$. The rate of hypoalbuminemia in the patients who died due to COVID-19 infection is higher than those who recovered from the disease $e^{5}$ Albumin down-regulates the expression of ACE-2 (Angiotensin Converting Enzyme) which is the main receptor of COVID-19 infection ${ }^{6}$. Among the COVID-19 patients, hypoalbuminemia was observed in $38.2 \%$ of patients who developed non-critical patients, $71.2 \%$ in critically ill patients, and $82.4 \%$ in the patients who developed mortality during hospitalization ${ }^{7}$. Anemia is an independent risk factor for severe COVID19 infection $^{8}$. The inflammatory response within the first 24 hours after admission to hospital in COVID-19 patients may be related to the severity of the disease'. This study aims to investigate the determinant role of serum inflammatory markers, albumin, and hemoglobin in predicting the diagnosis in patients with a pre-diagnosis of COVID-19 who are admitted to the emergency department.

\footnotetext{
${ }^{1}$ Canakkale Onsekiz Mart University, School of Medicine, Department of Emergency Medıcıne - Canakkale, Turkey.

${ }^{2}$ CanakkaleOnsekiz Mart University, School of Medicine, Department of Internal Medicine Division of Nephrology - Canakkale, Turkey.

*Corresponding author: drcananakman@gmail.com

Conflicts of interest: the authors declare there are no conflicts of interest. Funding: none.

Received on October 28, 2020. Accepted on December 10, 2020.
} 


\section{METHODS}

\section{Study Design}

Our study was planned retrospectively. The demographic findings, complete blood count, and serum biochemical values of the patients (pre-diagnosed with COVID-19) who were admitted to the emergency department of the Health, Practice and Research Hospital between March 31, 2020 and June 10, 2020 were analyzed. Our study started after the approval of the Local Ethics Committee (Date of Approval: 01.07.2020, $\mathrm{N}^{\circ}$ : 2020-09).

\section{Patients}

The information in the automation system was obtained retrospectively in the patients between the ages of 18 and 80 who were admitted to the emergency department with a pre-diagnosis of COVID-19 infection. Patients under the age of 18, patients with trauma, patients for whom sufficient information could not be obtained in the automation system were not included in the study. COVID-19 and non-COVID-19 patient groups were determined based on their nucleic acid test (PCR) results. All of the COVID (+) patients had pneumonia.

\section{Laboratory Analysis}

Serum creatinine, urea, and albumin analyses of the patients were examined with the colorimetric method on the Roche Cobas 6000 device 501 module. CRP (C-reactive protein) analyses were performed on the Cobas 6000 e501 module using a turbidimetric method, and complete blood count analyses were performed in the biochemistry laboratory using the electrical impedance method on the Beckman Coulter DXH 800 device.

In complete blood count, hemoglobin (Hb), leukocyte, mean platelet volume (MPV), neutrophil to lymphocyte ratio
(NLR), platelet to lymphocyte ratio (PLR), lymphocyte to monocyte ratio (LMR), monocyte to eosinophil (MER), neutrophil to monocyte ratio (NMR), red cell distribution width (RDW) and platelet parameters were analyzed.

\section{Statistics}

In data analysis, COVID (+) 48 (15.9\%) and COVID (-) 253 $(84.1 \%)$ patients were studied. The distribution of patients with and without the diagnosis of COVID (+) by gender was compared using chi-square analysis and the average age with the Mann-Whitney U Test (since age data did not show a normal distribution). MPV, NLR, PLR, leukocyte, Hb, platelet, RDW, LMR, NMR, MER, serum CRP, urea, creatinine, and albumin values of patients with and without COVID (+) diagnosis were compared using Mann-Whitney U Test (since the data did not show normal distribution). Serum albumin value was divided into two categories: 2.49-lower and 2.50-above. Then, the low and high albumin patient groups with and without COVID diagnosis were compared using the chi-square test. Does having a low or high serum albumin level an impact on whether being diagnosed with COVID or not? Analysis of this question was tested using Binary Logistic Regression in SPSS 19.0.

\section{RESULTS}

Three hundred and one patients ( 125 female and 176 male), 48 of whom were COVID (+) and 253 COVID (-), were included in the study. The average age of the patients was $54.76 \pm 20.8$. The comparison of the patients with COVID (+) and COVID $(-)$ according to gender and age were given in table 1 .

According to the results in table 1 , there was no significant relationship between whether or not diagnosed with COVID19 and gender distribution ( $p>0.05$ ). While $20 \%$ of the females

Table 1. The comparison of the patients with COVID (+) and COVID (-) according to gender and age.

\begin{tabular}{|c|c|c|c|c|c|c|c|c|c|}
\hline \multirow{2}{*}{\multicolumn{4}{|c|}{ Groups }} & \multicolumn{2}{|c|}{ Gender } & \multirow{2}{*}{ Total } & \multirow{2}{*}{$\chi^{2}$} & \multirow{2}{*}{ SD } & \multirow{2}{*}{$P^{*}$} \\
\hline & & & & \multirow{2}{*}{$\begin{array}{c}\text { Female } \\
99 \\
\end{array}$} & \multirow{2}{*}{$\begin{array}{c}\text { Male } \\
154 \\
\end{array}$} & & & & \\
\hline \multirow{3}{*}{\multicolumn{2}{|c|}{$\operatorname{COVID}(-)$}} & \multicolumn{2}{|c|}{$f$} & & & 253 & \multirow{6}{*}{2.794} & \multirow{6}{*}{1} & \multirow{6}{*}{0.095} \\
\hline & & \multicolumn{2}{|c|}{ for COVID diagnosis $\%$} & 39.1 & 60.9 & 100 & & & \\
\hline & & \multicolumn{2}{|c|}{ for Gender \% } & 79.8 & 87.0 & 84.1 & & & \\
\hline \multirow{3}{*}{\multicolumn{2}{|c|}{$\operatorname{COVID}(+)$}} & \multicolumn{2}{|c|}{ f } & 25 & 23 & 48 & & & \\
\hline & & \multicolumn{2}{|c|}{ for COVID diagnosis \% } & 52.1 & 47.9 & 100 & & & \\
\hline & & \multicolumn{2}{|c|}{ for Gender \% } & 20.2 & 13.0 & $15.9 \%$ & & & \\
\hline & & $\mathrm{n}$ & \multicolumn{3}{|c|}{ Mean (Standard Deviation) } & \multicolumn{3}{|c|}{ Median (Min-Max) } & $P * *$ \\
\hline \multirow{2}{*}{ Age } & COVID (-) & 253 & \multicolumn{3}{|c|}{$54.58(21.54)$} & \multicolumn{3}{|c|}{$57(19-92)$} & \multirow{2}{*}{0.878} \\
\hline & COVID (+) & 48 & \multicolumn{3}{|c|}{$55.69(16.81)$} & \multicolumn{3}{|c|}{$54(22-93)$} & \\
\hline
\end{tabular}

$P *$ : Chi-Square Test; $P * *$ : Mann Whitney U Test; SD: Standard deviation. 
were diagnosed with COVID-19, 80\% did not. While 13\% of the males were diagnosed with COVID-19, 87\% did not. On the other hand, $61 \%$ of 253 people with a diagnosis of COVID-19(-) were men and 39\% were women. Of the 48 people with a diagnosis of COVID-19(+), 52\% were female and $48 \%$ were male. There was no statistically significant difference between the groups in terms of age and gender ( $p>0.05)$.

Serum biochemistry and complete blood count values of the patients with COVID (+) and COVID (-) are shown in table 2. There was no statistically significant difference between the groups in terms of average serum albumin values $(\mathrm{p}=0.194)$. There was a statistically significant difference between the groups in terms of average hemoglobin, MPV, and MER ( $p$ values $0.029,0.009,0.008$, respectively). There was no statistically significant difference between the groups in terms of serum CRP, leukocyte, and NLR (p values $0.281,0.153$, 0.886 , respectively).

When the serum albumin cut-off value was determined as $2.5 \mathrm{~g} / \mathrm{dL}$, two subgroups were formed in the patients to be below and above this value. The comparison of patient subgroups

Table 2. The comparison of serum biochemistry and complete blood count values of the patients with COVID (+) and COVID (-).

\begin{tabular}{|c|c|c|c|c|c|}
\hline & & $\mathbf{N}$ & Mean (SD) & Median (Min-Max) & $P$ \\
\hline \multirow{2}{*}{ MPV } & COVID (-) & 253 & $8.59(1.016)$ & $8.5(6.7-12)$ & \multirow{2}{*}{0.009} \\
\hline & $\operatorname{COVID}(+)$ & 48 & $8.25(1.26)$ & $8.1(6.3-13.6)$ & \\
\hline \multirow{2}{*}{ NLR } & COVID (-) & 253 & $8.24(13.05)$ & $3.9(0.03-133.0)$ & \multirow{2}{*}{0.886} \\
\hline & COVID (+) & 48 & $6.95(7.89)$ & $4.4(0.70-39.5)$ & \\
\hline \multirow{2}{*}{ PLR } & COVID (-) & 253 & $24.19(40.53)$ & $12.9(2.30-470.0)$ & \multirow{2}{*}{0.524} \\
\hline & COVID (+) & 48 & $21.1(23.7)$ & $15.10(2.70-158.0)$ & \\
\hline \multirow{2}{*}{ Leukocyte $\left(\mathrm{mm}^{3}\right)$} & COVID (-) & 253 & $10196.4(4883.1)$ & $9200(2700-44300)$ & \multirow{2}{*}{0.153} \\
\hline & $\operatorname{COVID}(+)$ & 48 & $9481.2(4738.4)$ & 7750 (2600-20400) & \\
\hline \multirow{2}{*}{$\mathrm{Hb}(\mathrm{g} / \mathrm{dL})$} & COVID (-) & 253 & $13.21(2.21)$ & $13.4(3.30-17.80)$ & \multirow{2}{*}{0.029} \\
\hline & $\operatorname{COVID}(+)$ & 48 & $12.48(2.33)$ & $12.6(8.10-17.00)$ & \\
\hline \multirow{2}{*}{ Platelet $\left(\mathrm{mm}^{3}\right)$} & COVID (-) & 253 & $243.27(89.72)$ & $232(29-688)$ & \multirow{2}{*}{0.683} \\
\hline & $\operatorname{COVID}(+)$ & 48 & $254.7(106.4)$ & $232(47-523)$ & \\
\hline \multirow{2}{*}{ CRP (mg/dL) } & COVID (-) & 253 & $4.51(6.65)$ & $1.30(0.02-33.0)$ & \multirow{2}{*}{0.281} \\
\hline & $\operatorname{COVID}(+)$ & 48 & $6.37(8.93)$ & $1.55(0.07-31.40)$ & \\
\hline \multirow{2}{*}{ RDW } & COVID (-) & 253 & $14.66(2.10)$ & $14.10(12.10-24.90)$ & \multirow{2}{*}{0.176} \\
\hline & $\operatorname{COVID}(+)$ & 48 & $15.3(2.85)$ & $14.35(11.9-24.1)$ & \\
\hline \multirow{2}{*}{ LMR } & COVID (-) & 253 & $2.72(2.03)$ & $2.10(0.10-13.90)$ & \multirow{2}{*}{0.503} \\
\hline & COVID (+) & 48 & $2.32(1.32)$ & $1.90(0.30-5.80)$ & \\
\hline \multirow{2}{*}{ NMR } & COVID (-) & 253 & $13.25(17.55)$ & $8.90(0.90-190.20)$ & \multirow{2}{*}{0.608} \\
\hline & COVID (+) & 48 & $11.6(10.5)$ & $8.95(2.10-65.90)$ & \\
\hline \multirow{2}{*}{ MER } & COVID (-) & 253 & $17.77(29.49)$ & $7(0.10-173.0)$ & \multirow{2}{*}{0.008} \\
\hline & COVID (+) & 48 & $24.57(26.45)$ & $13.0(1.30-94.0)$ & \\
\hline \multirow{2}{*}{ Urea (mg/dL) } & COVID (-) & 253 & $38.84(38.64)$ & $29.50(8.60-528.0)$ & \multirow{2}{*}{0.136} \\
\hline & COVID (+) & 48 & $48.56(44.10)$ & $34.40(11.40-277.0)$ & \\
\hline \multirow{2}{*}{ Creatinine $(\mathrm{mg} / \mathrm{dL})$} & COVID (-) & 253 & $1.04(0.79)$ & $0.88(0.29-7.70)$ & \multirow{2}{*}{0.815} \\
\hline & $\operatorname{COVID}(+)$ & 48 & $1.14(0.88)$ & $0.86(0.45-5.89)$ & \\
\hline \multirow{2}{*}{ Albumin (g/dL) } & COVID (-) & 253 & $4.07(0.610)$ & $4.23(1.82-5.02)$ & \multirow{2}{*}{0.194} \\
\hline & $\operatorname{COVID}(+)$ & 48 & $3.88(0.77)$ & $4.18(2.17-5.05)$ & \\
\hline
\end{tabular}

SD: Standard deviation; P: Mann-Whitney U Test; MPV: mean platelet volume; NLR: neutrophil to lymphocyte ratio; PLR: platelet to lymphocyte ratio; RDW: red cell distribution width; LMR: lymphocyte to monocyte ratio; NMR: neutrophil to monocyte ratio; MER: monocyte to eosinophil. 
with low and high serum albumin values was shown in Table 3 . It was found that the patients with low serum albumin level $(<2.5 \mathrm{~g} / \mathrm{dL})$ were diagnosed $75 \%$ with COVID $(-)$, and $25 \%$ with COVID $(+)$. It was found that the patients with high serum albumin level $(>2.5 \mathrm{~g} / \mathrm{dL})$ were diagnosed $86.1 \%$ with COVID (-) and $13.9 \%$ with COVID (+). The effect of low or high serum albumin on COVID $(+)$ diagnosis is shown in table 3. Serum albumin level was found to be a statistically significant determinant in the diagnosis of COVID $(+)(\mathrm{p}<0.05)$. It was found that a high serum albumin level $(>2.5 \mathrm{~g} / \mathrm{dL})$ increased the diagnosis of COVID (-) approximately twice compared to being low $(<2.5 \mathrm{~g} / \mathrm{dL})(\operatorname{Exp}[\mathrm{B}]=2.069)$.

\section{DISCUSSION}

It is known that there is a relationship between hypoalbuminemia and severe COVID-19 infection ${ }^{3,4}$. Hypoalbuminemia has a negative impact on the morbidity and mortality caused by COVID-19 infection ${ }^{7}$. In acute infections, there is a rapid increase in albumin degradation at the cell level within hours ${ }^{10}$. Hypoalbuminemia is frequently encountered during COVID19 infection ${ }^{7}$. Serum albumin levels of severe COVID-19 cases were found to be lower than those with mild cases ${ }^{11}$. In our study, the average serum albumin level of COVID (+) patients was found to be lower than in the patient group with COVID $(-)$, but this is not a statistically significant difference. Serum albumin cut-off value was determined as $2.5 \mathrm{~g} / \mathrm{dL}$, and subgroups below (low) and above (high) were formed and analyzed again. Serum albumin level was found to be a determinant factor in the diagnosis of COVID (+). It was found that the group with high serum albumin level increased the diagnosis of COVID (-) approximately twice than the group with low levels. The constant term was found to be significant in the regression analysis. We can conclude from this finding that there may be another variable that affects the diagnosis of COVID-19 other than the serum albumin level taken into the regression equation.

In severe COVID-19 cases, $\mathrm{Hb}$ level was found to be lower than in patients with milder cases ${ }^{11,12}$ Anemia is an independent risk factor to severe COVID-19 infection ${ }^{8}$. Among the COVID-19 patients, Hb level was found to be lower in the patient group with comorbidity compared to the group without $^{13}$. In our study, Hb levels in the COVID (+) patient group were found to be statistically significantly lower than the COVID (-) group.

There are several studies in the literature comparing COVID (+) and COVID (-) patients in terms of complete blood count. It has been shown that in the patients diagnosed with COVID-19, the number of leukocytes and lymphocytes is lower than those with non-COVID-19 ${ }^{14}$. In another study, it was found that COVID-19 patients had lower leukocyte, lymphocyte, and eosinophil counts in complete blood count ${ }^{15}$. In a meta-analysis study, it was found that the platelet count is important for the diagnosis and prognosis of COVID-19 and the leukocyte and neutrophil count is a determinant factor, but high values reflect disease progression. In the same study, it was found that serum CRP levels in the patients with severe COVID-19 were not diagnostic ${ }^{16}$. In a study by Paliogiannis et al. ${ }^{17}$, COVID-19 patients had lower leukocyte, monocyte, and neutrophil counts and serum CRP levels compared to those with non-COVID-19 pneumonia. On the other hand, platelet count and MPV level were found to be

Table 3. Comparison of the patient groups with low $(<2.5 \mathrm{~g} / \mathrm{dL})$ and high $(>2.5 \mathrm{~g} / \mathrm{dL})$ serum albumin levels and the effect of low or high serum albumin on the diagnosis of COVID (+) (Binary Logistic Regression).

\begin{tabular}{|c|c|c|c|c|c|c|c|c|}
\hline \multirow{2}{*}{\multicolumn{2}{|c|}{ Groups }} & \multicolumn{2}{|c|}{ Albumin } & \multirow{2}{*}{ Total } & \multirow{2}{*}{$\chi^{2}$} & \multirow{2}{*}{ SD } & \multirow{2}{*}{$P *$} & \multirow{2}{*}{ Effect Size $(\phi)$} \\
\hline & & Low & High & & & & & \\
\hline \multirow{3}{*}{ COVID (-) } & $f$ & 42 & 211 & 253 & \multirow{6}{*}{4.207} & \multirow{6}{*}{1} & \multirow{6}{*}{0.040} & \multirow{6}{*}{0.118} \\
\hline & for COVID \% & $16.6 \%$ & $83.4 \%$ & $100.0 \%$ & & & & \\
\hline & for albumin \% & $75.0 \%$ & $86.1 \%$ & $84.1 \%$ & & & & \\
\hline \multirow{3}{*}{ COVID (+) } & $f$ & 14 & 34 & 48 & & & & \\
\hline & for COVID \% & $29.2 \%$ & $70.8 \%$ & $100.0 \%$ & & & & \\
\hline & for albumin \% & $25.0 \%$ & $13.9 \%$ & $15.9 \%$ & & & & \\
\hline \multirow{2}{*}{\multicolumn{2}{|c|}{ Serum albumin $>2.5 \mathrm{~g} / \mathrm{dL}$}} & B & S.E. & Wald & & df & Sig & $\operatorname{Exp}(B)$ \\
\hline & & 727 & 360 & 4.084 & & 1 & 043 & 2.069 \\
\hline \multicolumn{2}{|l|}{ Invariant } & -1.825 & 185 & 97.579 & & 1 & 000 & 161 \\
\hline
\end{tabular}

P*: Chi-Square Test, Reference Group: COVID (+). SD: standard deviation; B:beta; SE:standard error; df:degree of freedom; Sig.:significant; Exp (B): expected beta. 
high. Differently, in the study conducted by Djakpo et al. ${ }^{13}$, no difference was found between COVID-19 patients and non-COVID-19 patients in terms of leukocyte, lymphocyte, and platelet values in complete blood count. In the same study, serum CRP levels were found to be statistically significantly higher in COVID-19 patients. In our study, a difference was observed between the COVID (+) and COVID (-) groups in terms of MPV and MER.MPV is lower in the COVID (+) group compared to COVID (-) group, and the MER was found to be high. There was no difference between the groups in terms of leukocyte, platelet, and serum CRP levels.

MER, NLR, and PLR are some of the inflammation parameters that play a key role in inflammatory oncological and cardiovascular diseases ${ }^{18,19}$. In our study, no difference was found between COVID (+) and COVID (-) groups in terms of NLR and PLR, but MER was found to be higher in the COVID (+) group, and the difference was statistically significant. In a meta-analysis study, NLR was found to be higher in severe COVID-19 patients compared to non-COVID patients ${ }^{20}$. In a study by Qu et al. ${ }^{21}$, while the PLR level of severe COVID-19 patients during admission to the hospital was similar to those of COVID patients who did not have severe disease, this situation changed during the period of the platelet peak and the PLR rate increased in severe cases. Our study is observational and cross-sectional and based on the laboratory data of COVID-19 patients at the time of their admission to the emergency department. Therefore, biochemical data of the patients' follow-up in the service or intensive care unit were not used in the study.
There were several limitations of our study. Since it was a single-center study, the number of COVID-19 (+) patients was low. Complete blood count and serum biochemistry values of the patients at the time of admission to the emergency department were studied. The values of the hospitalized patients in the service and intensive care unit were not included in the study.

In conclusion, high serum albumin level $(>2.5 \mathrm{~g} / \mathrm{dL})$ increases COVID (-) diagnosis approximately twice as compared to low $(<2.5 \mathrm{~g} / \mathrm{dL})$. In COVID $(+)$ patients, Hb level is lower than in COVID (-) patients. MER and MPV could be a new indicator that predicts COVID-19 infection.

\section{Main points}

- Serum albumin level is a statistically significant determinant in the diagnosis COVID (+).

- High serum albumin level $(>2.5 \mathrm{~g} / \mathrm{dL})$ increases COVID (-) diagnosis approximately twice as compared to low $(<2.5 \mathrm{~g} / \mathrm{dL})$.

- MER and MPV could be a new indicator that predicts COVID-19 infection.

- In COVID (+) patients, $\mathrm{Hb}$ level is lower than in COVID (-) patients.

\section{AUTHORS' CONTRIBUTIONS}

CA: Conceptualization, Data Curation, Formal Analysis, Writing - Original Draft, Writing - Review \& Editing. SB: Conceptualization, Data Curation, Formal Analysis, Writing - Original Draft, Writing - Review \& Editing.

\section{REFERENCES}

1. Wang C, Horby PW, Hayden FG, Gao GF. A novel coronavirus outbreak of global health concern. Lancet. 2020;395(10223):470-3. https://doi.org/10.1016/S01406736(20)30185-9

2. Lai CC, Shih TP, Ko WC, Tang HJ, Hsueh PR. Severe acute respiratory syndrome coronavirus 2 (SARS-CoV-2) and coronavirus disease-2019 (COVID-19): the epidemic and the challenges. Int J Antimicrob Agents. 2020;55(3):105924. https://doi.org/10.1016/j.ijantimicag.2020.105924

3. Aziz M, Fatima R, Lee-Smith W, Assaly R. The association of low serum albumin level with severe COVID-19: a systematic review and meta-analysis. Crit Care. 2020;24(1):255. https:// doi.org/10.1186/s13054-020-02995-3

4. Huang J, Cheng A, Kumar R, Fang Y, Chen G, Zhu Y, et al. Hypoalbuminemia predicts the outcome of COVID-19 independent of age and co-morbidity. J Med Virol. 2020;92(10):2152-8. https://doi.org/10.1002/jmv.26003
5. Chen T, Wu D, Chen H, Yan W, Yang D, Chen G, et al. Clinical characteristics of 113 deceased patients with coronavirus disease 2019: retrospective study. BMJ. 2020;368:m1091. https://doi.org/10.1136/bmj.m1091

6. Liu BC, Gao J, Li Q, Xu LM. Albumin caused the increasing production of angiotensin II due to the dysregulation of ACE/ ACE2 expression in HK2 cells. Clin Chim Acta. 2009;403(12):23-30. https://doi.org/10.1016/j.cca.2008.12.015

7. Huang W, Li C, Wang Z, Wang H, Zhou N, Jiang J, et al. Decreased serum albumin level indicates poor prognosis of COVID-19 patients: hepatic injury analysis from 2,623 hospitalized cases. Sci China Life Sci. 2020;63(11):1678-87. https://doi.org/10.1007/s11427-020-1733-4

8. Tao Z, Xu J, Chen W, Yang Z, Xu X, Liu L, et al. Anemia is associated with severe illness in COVID-19: A retrospective cohort study. J Med Virol. 2021;93(3):1478-88. https://doi. org/10.1002/jmv.26444 
9. Zeng Z, Yu H, Chen H, Qi W, Cehn L, Chen G, et al. Longitudinal changes of inflammatory parameters and their correlation with disease severity and outcomes in patients with COVID-19 from Wuhan, China. Crit Care. 2020;24(1):525. https://doi. org/10.1186/s13054-020-03255-0

10. Bahar M. Hipoalbumineminin klinik açıdan değerlendirilmesi. Ankem Dergis [Internet]. 2002;16(3):356-9. Available from: https://www.ankemdernegi.org.tr/ANKEMJOURNALPDF/ ANKEM_16_3_356_359.pdf

11. Danwang C, Endomba FT, Nkeck JR, Wouna DLA, Robert A, Noubiap JJ. A meta-analysis of potential biomarkers associated with severity of coronavirus disease 2019 (COVID-19). Biomark Res. 2020;8:37. https://doi.org/10.1186/s40364-020-00217-0

12. Taneri PE, Gómez-Ochoa SA, Llanaj E, Raguindin PF, Rojas LZ, Roa-Díaz ZM, et al. Anemia and iron metabolism in COVID19: a systematic review and meta-analysis. Eur J Epidemiol. 2020;35(8):763-73. https://doi.org/10.1007/s10654-020-00678-5

13. Djakpo DK, Wang Z, Zang R, Chen X, Chen P, Antoine MMLK. Blood routine test in mild and common 2019 coronavirus (COVID-19) patients. Biosci Rep. 2020;40(8):BSR20200817. https://doi.org/10.1042/BSR20200817

14. Luo L, Luo Z, Jia Y, Zhou C, He J, Lyu J, et al. CT differential diagnosis of COVID-19 and non-COVID-19 in symptomatic suspects: a practical scoring method. BMC Pulm Med. 2020;20(1):129. https://doi.org/10.1186/s12890-020-1170-6

15. Li YX, Wu W, Yang T, Zhou W, Fu YM, Feng QM, et al. Characteristics of peripheral blood leukocyte differential counts in patients with COVID-19. Zhonghua Nei Ke Za Zhi. 2020;59(5):372-4. https:// doi.org/10.3760/cma.j.cn112138-20200221-00114
16. Soraya GV, Ulhaq ZS. Crucial laboratory parameters in COVID-19 diagnosis and prognosis: an updated meta-analysis. Med Clin (Barc). 2020;155(4):143-51. https://doi.org/10.1016/j. medcli.2020.05.017

17. Paliogiannis P, Zinellu A, Scano V, Mulas G, De Riu G, Pascale $\mathrm{RM}$, et al. Laboratory test alterations in patients with COVID-19 and non COVID-19 interstitial pneumonia: a preliminary report. J Infect Dev Ctries. 2020;14(7):685-90. https://doi. org/10.3855/jidc. 12879

18. Nishida $Y$, Hosomi S, Yamagami H, Yukawa T, Otani K, Nagami $Y$, et al. Neutrophil-to-lymphocyte ratio for predicting loss of response to infliximab in ulcerative colitis. PLoS One. 2017;12(1):e0169845. https://doi.org/10.1371/journal. pone. 0169845

19. Imtiaz F, Shafique K, Mirza SS, Ayoob Z, Vart P, Rao S. Neutrophil lymphocyte ratio as a measure of systemic inflammation in prevalent chronic diseases in Asian population. Int Arch Med. 2012;5(1):2. https://doi. org/10.1186/1755-7682-5-2

20. Ghahramani S, Tabrizi R, Lankarani KB, Kashani SMA, Rezaei S, Zeidi $N$, et al. Laboratory features of severe vs. non-severe COVID-19 patients in Asian populations: a systematic review and meta-analysis. Eur J Med Res. 2020;25(1):30. https://doi.org/10.1186/s40001-02000432-3

21. Qu R, Ling Y, Zhang YH, Wei LY, Chen $X$, Li XM, et al. Plateletto-lymphocyte ratio is associated with prognosis in patients with coronavirus disease-19. J Med Virol. 2020;92(9):1533-41. https://doi.org/10.1002/jmv.25767 